\title{
Novel foamy origin for singlet fermion masses
}

\author{
John Ellis, ${ }^{1,2}$ Nick E. Mavromatos, ${ }^{1}$ and Dimitri V. Nanopoulos ${ }^{3}$ \\ ${ }^{1}$ Theoretical Particle Physics and Cosmology Group, Department of Physics, King's College London, \\ Strand WC2R 2LS London, United Kingdom \\ ${ }^{2}$ Theoretical Physics Department, CERN, CH-1211 Geneva 23, Switzerland \\ ${ }^{3}$ George P. and Cynthia W. Mitchell Institute for Fundamental Physics and Astronomy, \\ Texas A \& M University, College Station, Texas 77843, USA; \\ Astroparticle Physics Group, Houston Advanced Research Center (HARC), \\ Mitchell Campus, Woodlands, Texas 77381, USA; \\ Division of Natural Sciences, Academy of Athens, Athens 106 79, Greece
}

(Received 24 June 2017; published 17 October 2017)

\begin{abstract}
We show how masses for singlet fermions can be generated by interactions with a D-particle model of space-time foam inspired by brane theory. It has been shown previously by one of the authors (N. E. M.) that such interactions may generate dynamically small masses for charged fermions via the recoils of D-particle defects interacting with photons. In this work we consider the direct interactions of D-particle with uncharged singlet fermions such as right-handed neutrinos. Quantum fluctuations of the lattice of D-particles have massless vector (spin-one) excitations that are analogues of phonons. These mediate forces with the singlet fermions, generating large dynamical masses that may be communicated to light neutrinos via the seesaw mechanism.
\end{abstract}

DOI: 10.1103/PhysRevD.96.086012

\section{INTRODUCTION AND MOTIVATION}

It is widely accepted that neutral singlet particles with no symmetries to protect them, such as right-handed neutrinos, will in general have very large masses that might approach the Planck mass at which quantum-gravitational effects become important. It has long been argued that quantummechanical effects generate a foamy structure of space-time on small scales [1], interactions with which might have observable implications for the propagation of particles [2]. Since foam constitutes a fluctuating background, it is not obvious whether the conventional rules of quantum field theory apply [3], and one possibility is that interactions with the foam might induce Lorentz- [4] and/or CPTviolating [5,6] dispersion relations for photons [2] and gravitons [7].

Here we investigate the more conservative possibility that interactions with space-time foam might contribute to the large masses expected for neutral singlet particles.

We have long advocated a toy model of space-time foam, motivated by string theory [8-12], in which the foamy space-time structures are provided by stringy defects, Dparticles, that interact with matter particles described by string excitations on branes. These fluctuating D-foam defects break Poincaré invariance, and their recoil during

Published by the American Physical Society under the terms of the Creative Commons Attribution 4.0 International license. Further distribution of this work must maintain attribution to the author(s) and the published article's title, journal citation, and DOI. the interaction with propagating string states breaks Lorentz invariance. The nontrivial transfer of momentum during the interactions of matter strings with D-foam defects is mediated by the emission of nonlocal intermediate string states that do not admit a local effective action description, leading to a violation of Lorentz invariance that is subject to probes using astrophysical sources. The ultraviolet completion of this type of foam model is provided by string/brane theory itself [13].

It was argued in [14] that another observable implication of D-foam would be the dynamical generation of small nonperturbative masses for charged fermions. This was the result of Lorentz-violating higher-derivative terms in the Maxwell action induced by the interaction of photons with the D-particles, which in turn are communicated to the charged sector via the coupling with the electromagnetic field. ${ }^{1}$ The effective field theory model upon which this effect is based, onto which the D-foam/fermion/photon effective action is mapped at low energies, ignoring the nonlocal stringy structures of the D-particles, has been studied in a different context in [15].

It was argued in [14] that the quantum fluctuations of the D-particle defects in target space lead to a novel correspondence principle, through which an antisymmetric tensor background field in phase space, representing the recoil velocity of the defect during its interaction with matter, is mapped [16] into a spatial derivative operator

\footnotetext{
${ }^{1}$ Due to charge conservation, a direct coupling of a charged fermion to the D-foam excitations is forbidden by the electromagnetic U(1) gauge symmetry [10].
} 
along the direction of the recoil. In this way, the resulting Finsler-type [17] Born-Infeld (FBI) Lagrangian that describes the low-energy dynamics of open strings on a brane world in interaction with the D-particles may be transformed into an effective Lagrangian with Lorentzviolating higher-order spatial-derivative terms, reproducing the minimal Lorentz-violating modification of quantum electrodynamics (QED) considered in [15]. It was further argued that this model provides a novel way of generating charged-particle masses dynamically. We should stress that this model is not of Horava-Lifshitz type [18], in the sense that there is no anisotropic scaling between time and space coordinates. The Lorentz violation is manifested through higher-order spatial-derivative terms that respect rotational symmetry in three-space, but are suppressed by an effective mass scale. The presence of this scale and the Lorentzviolating terms generate dynamically small masses for charged fermions, for arbitrarily weak gauge fields.

Our link [14] of such a Lagrangian with D-foam, and ultimately with more general models of quantumgravitational foam, constitutes an explicit realization of the effects of a foam medium in slowing down some particles via mass generation. In our case, the mass scale that suppresses the Lorentz-violating terms is expressed in terms of the string mass scale and fundamental parameters of the foam such as the variance of its quantum fluctuations. There are issues associated with a quantum-ordering ambiguity in our construction, which was argued in [14] to be resolved by appealing to the important role of the dynamical mass generation in curing infrared instabilities in the model. In this way, a selection of the physically relevant class of quantum orderings could be made, resulting [14] in the above-mentioned mapping of the quantum-ordered FBI effective action onto the QED action of [15] at low energies. Any further ordering ambiguities within this class of models are absorbed into the quantum fluctuation parameters of the D-foam, which are regarded as phenomenological at this stage.

In this paper we extend this model of D-foam and its interactions with stringy matter to show how they may generate large masses for neutral singlet fermions such as right-handed neutrinos via spin-one excitations of the space-time foam that are analogous to phonons in condensed-matter physics. If the singlet fermions are Dirac, we find that the foam interactions can be described by the minimal Lorentz-violating effective action of [15], whereas if the singlet fermions are Majorana, as might be the case for right-handed neutrinos, they can be described by the Lorentz-violating low-energy effective action of [19].

The structure of the article is as follows: in Sec. II we review the mapping of the low-energy dynamics of D-foam onto an appropriately quantum-ordered Finsler-Born-Infeld action, which yields the minimal Lorentz-violating QEDtype model of [15,19], following the procedure of [14]. The novelty in the dynamical generation of masses for neutral singlet fields, as compared to the charged fermion case considered in [14], is that the quantum fluctuations of the D-particles themselves [which are represented by vector (spin-one) quantum fields] couple directly to the singlet fields via minimal couplings that resemble the corresponding QED fermion-photon coupling. However, this vector field is not a photon, nor is it a conventional gauge field if the singlet fields are Majorana fermions, as is often assumed in quantum field theory models. We discuss in Sec. III how this coupling generates a dynamical mass for the singlet fermions in this model, expressing the obtained masses in terms of parameters of the D-foam model, such as the string scale, the density of D-foam excitations and the variance of the quantum fluctuations. Some phenomenological issues related to the sizes of the proposed masses, and the possibility of their enhancement in some multibrane-world scenarios, are discussed briefly in Sec. IV. Our conclusions and an outlook are presented in Sec. V.

\section{A MODEL FOR D-FOAM INTERACTIONS WITH SINGLET FERMIONS}

In this section we discuss an effective field theory model for the Lorentz-violating vector interactions of a singlet fermion with quantum-fluctuating D-particles. We demonstrate that Lorentz-violating Lagrangians of the form discussed in [15] may arise as (parts of) the low-energy, weak-field limit of an effective Born-Infeld Lagrangian describing the propagation of vector bosons $\mathcal{A}_{\mu}$ that describe the quantum fluctuations of the D-particle foam. We assume for simplicity a uniform lattice of D-particle defects that puncture a three-dimensional brane world (D3-brane), through which the singlet fermions propagate. The vector $\mathcal{A}_{\mu}$ is the low-energy mode of open strings stretched between the D-particle and the D3-brane world and, like the phonons in condensed-matter physics, it plays the role of a Goldstone boson. However, it is a vector boson, as a result of the spontaneous breaking of translational invariance by fluctuating D-particle defects in the D-foam vacuum.

As already mentioned, such models have been termed D-particle foam $[8,9,11,12]$, and have a variety of applications, ranging from providing microscopic stringinspired models of Lorentz-violating vacuum refractive indices $[11,12,20]$ to enhancing the abundance of thermal dark matter relics, with interesting implications for astroparticle phenomenology [21].

\section{A. Features of the D-foam model}

The basic idea of the D-foam model is shown in Fig. 1. A (possibly compactified from higher dimensions) three-brane world is moving in a higher-dimensional bulk space, that is punctured by D-particles. Depending on the string theory considered, the latter can be either pointlike 
D0-branes (as in Type IIA strings) [8,9,11] or D3-branes wrapped around appropriate three cycles (as in Type IIB strings [12]). As the brane world and the D-particles move in the bulk, they may encounter each other and an observer on the D3-brane sees the D-particle defects as flashing on and off. In these scenarios, ordinary matter and radiation are represented by open strings with their ends attached on the D3-brane. ${ }^{2}$

Gravitational degrees of freedom and singlet fermions are allowed to propagate in the bulk [22], in contrast to Standard Model (SM) fields, which are confined to the brane. However, when the bulk space is compactified, e.g., through periodic boundary conditions imposed on various brane worlds that populate the bulk, see Fig. 1, these fermions acquire Kaluza-Klein masses that are suppressed by the size of the extra bulk dimension. This mechanism can generate Majorana masses, e.g., for right-handed neutrinos $[22,23]$ in brane world scenarios. Here we assume that such a mechanism either is not in operation, because our singlet fermions are also confined on the brane world, like the SM excitations, or that the size of the extra dimensions are sufficiently large that the Kaluza-Klein generated masses are negligible. In this case, the dominant source of mass for the singlet fermions may be their interaction with the foam.

Indeed, there are nontrivial interactions of D-particles with open strings, provided there is no electric flux along the open string excitations, i.e., provided that the string states are electrically neutral. ${ }^{3}$ This is because the electrically neutral D-particles can "cut" an open string, leading to the emission of intermediate strings stretched between the D-particle and the D3 brane [11,20]. If the open string state carries electric flux, such a cutting procedure is not

\footnotetext{
${ }^{2}$ As clarification for the reader, we emphasize that we consider the set up of [9], which involves D8 branes interacting with bulk D0-branes, only as a concrete example for the ideas in this work. We do not consider D0, D3, and D8 branes simultaneously. Within the context of Type IIA strings and the corresponding lowenergy supergravity theory [9] that we focus on here, we consider only D8 and D0 branes, viewing our D3 brane world as arising from compactification of D8 branes. We do not consider details of such compactifications, as our purpose here is to discuss some generic features of the (recoil) effects of D-particles on matter singlet fermions on the brane worlds, and refer to [9] for details of the dynamics of D0 and D8 branes. Within Type IIA theory, in which D4 and D6 branes are allowed, D3 brane worlds may arise as D4 or D6 branes wrapped around appropriate cycles or compactified in more complicated manifolds, but we assume that their dynamics is not important for the generic features of the dynamical mechanism for mass generation for singlet fermions we discuss here.

${ }^{3}$ In the case of Type IIB strings, where the D-particles are not pointlike, there may be interactions between electrically charged excitations and the D-particles, but the foam effects on such charged particles are suppressed [12] compared to those on neutral particles. Therefore, in our discussion below we do not differentiate between these two cases, except when we discuss some phenomenological issues in Sec. IV.
}

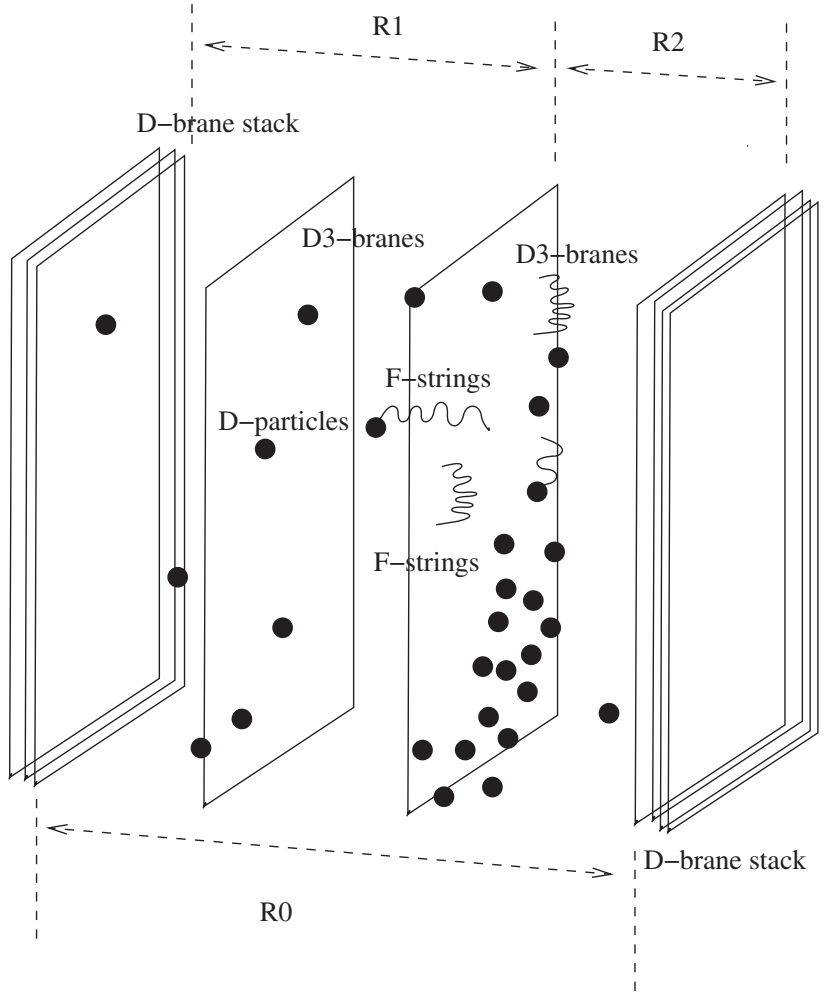

FIG. 1. Schematic representation of the D-particle model of space-time foam proposed in [9]. There are two stacks of D8-branes, each attached to an orientifold plane, whose special reflective properties enable them to provide a natural compactification of the bulk dimension, and the bulk is punctured by D0-branes (D-particles). An isolated D-particle cannot exist in the absence of a D-brane, because of gauge flux conservation. Standard Model matter is represented by pen strings on the brane world, whereas gravitons and singlet fermions can propagate in the bulk. The latter can interact directly with the D-particles in a topologically nontrivial way, involving cutting and splitting the open string representing the singlet fermion.

allowed, due to charge conservation. As a result of the local SU(2) gauge symmetry of lepton doublets, this also precludes light (active) neutrinos from interacting directly with the foam. However, singlet fermions such as righthanded neutrinos may interact directly with the D-particles as can photons and gravitons.

As discussed in [20], the first-quantization picture for the interaction of an open-string state such as a photon with a $\mathrm{D}$-particle is provided by a world-sheet $\sigma$-model with the following deformation:

$\mathcal{V}_{\text {recoil velocity part }}^{\text {implese }}=\frac{1}{2 \pi \alpha^{\prime}} \sum_{i=1}^{D} \int_{\partial D} d \tau u_{i} X^{0} \Theta\left(X^{0}\right) \partial_{n} X^{i}$,

where $M_{s}$ is the string (mass) scale, $g_{s}$ is the string coupling, and $u_{i}$ is the recoil velocity of the D-particle. The latter is assumed to be heavy, and $u^{i}$ is the spatial part of the four-velocity of the D-particle, which is well 
approximated by the ordinary velocity, to leading order for nonrelativistic, slow-moving, heavy D-particles. The limit $D$ in the sum in (2.1) denotes the appropriate number of spatial target-space dimensions, which is $D=3$ for a recoiling D-particle confined on a D3 brane, as is our case here. The operator $\Theta_{\varepsilon}\left(X^{0}\right)=-i \int_{-\infty}^{\infty} \frac{d \omega}{\omega+i \varepsilon}: \varepsilon \rightarrow 0^{+}$, is a regularized Heaviside world-sheet operator.

The recoil operators satisfy $[16,24]$ a specific type of conformal algebra, termed a logarithmic conformal algebra [25]. This algebra is the limiting case of world-sheet algebras that can still be classified by conformal blocks. The impulse operator $\Theta\left(X^{0}\right)$ is regularized so that the logarithmic conformal field theory algebra is respected. ${ }^{4}$ The conformal algebra is consistent with momentum conservation during recoil [16,24], which yields the following expression for the recoil velocity $u_{i}$ in terms of momentum transfer during the scattering:

$$
u_{i}=g_{s} \frac{p_{1}-p_{2}}{M_{s}}
$$

with $M_{s} / g_{s}$ being the D-particle "mass" and $\Delta p \equiv p_{1}-p_{2}$ the momentum transferred to the string state by its scattering with the D-particle.

We note next that one can write the boundary recoil/ capture operator (2.1) as a total derivative over the bulk of the world-sheet, by means of the two-dimensional version of Stokes theorem. Omitting from now on the explicit summation over repeated $i$-indices, which is understood to be over the spatial indices of the D3-brane world, we then write

$$
\begin{aligned}
& \mathcal{V}_{\text {recoil velocity part }}^{\text {impulse }} \\
& \quad=\frac{1}{2 \pi \alpha^{\prime}} \int_{D} d^{2} z \epsilon_{\alpha \beta} \partial^{\beta}\left(\left[u_{i} X^{0}\right] \Theta_{\varepsilon}\left(X^{0}\right) \partial^{\alpha} X^{i}\right) \\
& \quad=\frac{1}{4 \pi \alpha^{\prime}} \int_{D} d^{2} z\left(2 u_{i}\right) \epsilon_{\alpha \beta} \partial^{\beta} X^{0}\left[\Theta_{\varepsilon}\left(X^{0}\right)+X^{0} \delta_{\varepsilon}\left(X^{0}\right)\right] \partial^{\alpha} X^{i},
\end{aligned}
$$

where $\delta_{\varepsilon}\left(X^{0}\right)$ is an $\varepsilon$-regularized $\delta$-function.

We consider relatively large times after the after the moment of impulse, $X^{0}=0$, at which the initial open-string state splits into intermediate open strings as a result of the topologically nontrivial interactions with the D-particle. For the phenomenological purposes of this work, the expression (2.3) is equivalent to a deformation describing an open string propagating in an antisymmetric $B_{\mu \nu}{ }^{-}$ background ( $B$-field) corresponding to a constant external "electric" field in target-space:

\footnotetext{
${ }^{4}$ This can be done by using the world-sheet scale, $\varepsilon^{-2} \equiv \ln (L / a)^{2}$, with $a$ an ultraviolet scale and $L$ the worldsheet area, as a regulator [16,24]: $\Theta_{\varepsilon}\left(X^{0}\right)=-i \int_{-\infty}^{\infty} \frac{d \omega}{\omega-i \varepsilon} e^{i \omega X^{0}}$, taking the quantity $\varepsilon \rightarrow 0^{+}$at the end of the calculations.
}

$$
T^{-1} B_{i 0}=-T^{-1} B_{0 i}=u_{i}=\frac{g_{s} \Delta p_{i}}{M_{s}}, \quad T=\frac{1}{2 \pi \alpha^{\prime}},
$$

where $T$ denotes the (open) string tension, 0 is a temporal index, and $i$ is a spatial index. The reader should notice here the phase-space dependence of the background field, which resembles an "electric" field background but in momentum space [20], and is therefore of Finsler type [17].

In the above analysis we have ignored a possible angular momentum operator, which may also arise as a result of the nontrivial scattering of a photon on a D-particle defect. At the $\sigma$-model level, the latter is also described by a logarithmic conformal algebra deformation that, for the three-brane case to which we restrict our attention here, takes the form:

$$
\begin{aligned}
V_{\text {ang momD-part }}^{\text {impulse }}= & T^{-1} \int_{\partial \Sigma} u^{i} \epsilon_{i j k} X^{j} \Theta_{\varepsilon}\left(X^{0}\right) \partial_{n} X^{k} \\
= & T^{-1} \int_{\Sigma} \varepsilon^{\alpha \beta}\left(u^{i} \epsilon_{i j k} \Theta_{\varepsilon}\left(X^{0}\right) \partial_{\alpha} X^{j} \partial_{\beta} X^{k}\right. \\
& \left.+u^{i} \epsilon_{i j k} X^{j} \delta_{\varepsilon}\left(X^{0}\right) \partial_{\alpha} X^{0} \partial_{\beta} X^{k}\right)
\end{aligned}
$$

where we have again applied the two-dimensional Stokes theorem, $\alpha, \beta=1,2$ are world-sheet indices, and $\epsilon^{\alpha \beta}$ is the world-sheet Levi-Civita tensor, and $\epsilon_{i j k}$ is the antisymmetric symbol in the three spatial dimensions of the brane world. The logarithmic conformal properties [24] of the deformation arise from the $X^{j}$ parts. For the relatively large times $X^{0}>0$ after the impulse that we consider here, we may ignore the $\delta$-function terms, and in this case the effects of the angular momentum deformation in target space are equivalent to the open string propagating in an antisymmetric tensor "magnetic-field" type background with spatial components given by

$$
T^{-1} B_{i j}=-T^{-1} B_{j i}=\epsilon_{i j k} u^{k}
$$

This should be combined with (2.4) in order to provide a complete description of the averaged interactions of a photon with the D-foam, in a first-quantized version.

The quantity $u_{i}$ in (2.4), which involves the momentum transfer, $\Delta p_{i}$, can be modeled by a local operator using the following parametrization [26]:

$$
u_{i}=g_{s} \frac{\Delta p_{i}}{M_{s}}=\frac{g_{s}}{M_{s}} r_{i} p_{i}, \quad \text { no sum over } i=1,2,3,
$$

where the (dimensionless) variables $r_{i}, i=1,2,3$, appearing above are related to the fraction of momentum that is transferred in collision with a D-particle in each spatial direction $i$. In the stochastic foam approximation [26] these parameters are taken as Gaussian normal random variables with a range $-\infty$ to $+\infty$ and defining moments 


$$
\begin{gathered}
\left\langle r_{i}\right\rangle=0, \\
\left\langle r_{i} r_{j}\right\rangle=0, \quad \text { if } i \neq j
\end{gathered}
$$

and

$$
\sigma_{i}^{2}=\left\langle r_{i}^{2}\right\rangle-\left\langle r_{i}\right\rangle^{2}=\left\langle r_{i}^{2}\right\rangle \neq 0 .
$$

We assume that the foam is isotropic, which implies that $r_{i}=r$ for all $i=1,2,3$. In this case the variances

$$
\left\langle\left(r_{i}\right)^{2}\right\rangle=\sigma^{2}, \quad i=1,2,3,
$$

are equal in all spatial directions.

However, for full rotational invariance in three space of the Born-Infeld action (2.15), involving interactions between the velocity fields $u_{i}$ and the Maxwell tensor $F^{\mu \nu}$ of the vector field $\mathcal{A}_{\mu}$, one more requirement is necessary. When considering the application of the above averages to the recoil velocities, the latter must take the form:

$$
\left\langle u_{i}\right\rangle=0, \quad\left\langle u_{i} u_{j}\right\rangle=\delta_{i j} \frac{\sigma^{2} g_{s}^{2}}{M_{s}^{2}} p^{k} p_{k}
$$

The averages $\langle\cdots\rangle$ denote both statistical averages over populations of D-particle defects in the foam, cf. Fig. 1, as well as target-space quantum fluctuations. The latter can be induced by considering the summation over world-sheet genera [16]. This is quite important for our purposes, and we return later to this issue and its implications.

For the moment, we remark that the stochasticity conditions (2.8) imply the restoration of Lorentz invariance in the statistical mean, with nontrivial fluctuations described in the isotropic and three-space rotationally invariant case by (2.9), (2.11), and (2.12). Hence, although Lorentz invariance and spatial isotropy is lost locally in individual scatterings of photons with D-particles, due to the presence of the recoil velocity of the D-particle, $u_{i}$, the isotropy of the foam washes such violations out on average, and isotropy and rotational invariance are restored.

\section{B. Finsler-Born-Infeld (FBI) effective actions}

We turn now to the singlet fermion sector. Unlike the charged fermions of [14], here, in principle, there are recoilinduced contributions, since a singlet fermion can interact directly with the D-brane, causing recoil of the latter. Since the recoil is described by the antisymmetric tensor field excitations $B_{\mu \nu}$ in a $\sigma$-model framework, string theory gives specific rules for coupling this background to fermionic excitations propagating in the bulk. Any bulk world-sheet action would involve the field strength of $B_{\mu \nu}$, namely $H_{\mu \nu \rho}=\partial_{[\mu} B_{\nu \rho]}$, where $[\ldots]$ denotes total antisymmetrization of the indices. Such field strength terms are dictated by an Abelian gauge symmetry $B_{\mu \nu} \rightarrow B_{\mu \nu}+\partial_{[\mu} \Lambda_{\nu]}$ that characterizes the closed-string sector describing the gravitational multiplet of the string, to which the antisymmetric tensor belongs. A minimal string theory coupling between fermions and the $H$-field strength would be of the form [13]:

$$
\int d^{4} x \epsilon_{\mu \nu \rho \sigma} H^{\nu \rho \sigma} \bar{\psi} \gamma \gamma^{\mu} \gamma^{5} \psi
$$

where $\epsilon_{\mu \nu \rho \sigma}$ is the covariant Levi-Civita tensor and $\gamma^{5} \psi=\psi$ for right-handed fermions $\psi$. In principle, a nonzero $H$ field strength could arise in our model from inhomogeneous foam situations, in which the variance (2.11) would depend on space-time. In the approximation of a homogeneous foam that is appropriate in the current epoch of our Universe that is of interest in the present article, we can neglect any such dependence on the variances (2.12), and thus set to zero couplings of the form (2.13).

We recall that the recoil velocity $u_{i}$ refers to components of the bulk D-particle velocity parallel to the brane worlds. There are also components of the velocity perpendicular to the brane worlds. These generate forces between Dparticles and brane worlds that they cross, due to fundamental strings created in such processes, as discussed in detail in [27] and used in [9]. Such strings are sources of Neveu-Schwarz flux three-forms on the three-brane with world-volume field strengths $H_{\mu \nu \rho}^{\prime}, \mu, \nu, \rho=0, \ldots 3$ [where the prime indicates that they are distinct from the corresponding recoil fields appearing in (2.13)]. As demonstrated in [9], these processes make contributions to the vacuum energy on the brane, which are assumed to be suppressed by opposite-sign contributions to the vacuum energy density due to other bulk D-particles that lie more than a few string lengths away from the brane world, or other vacuum energy contributions on the brane due to quantum field excitations. Despite the fact that the singlet fermions are allowed to propagate in the bulk, once they are on the brane world, as we consider here, they couple to $H_{\mu \nu \rho}^{\prime}$ via terms of the form (2.13). Such couplings can be integrated out exactly in a $(3+1)$-dimensional path integral of the brane world effective action. The corresponding kinetic terms of the $H^{\prime}$ field on the brane are (to lowest order in derivatives) [13]: $\int d^{4} x \sqrt{-g} \frac{1}{12} H_{\mu \nu \rho}^{\prime} H^{\prime \mu \nu \rho}$. Integrating out exactly the $H$-terms in the brane world path integral yields repulsive four-fermion interactions of the type

$$
\int d^{4} x \sqrt{-g} \frac{3}{16} \kappa^{2} J_{\mu}^{5} J^{5 \mu}, \quad J^{5 \mu}=\bar{\psi} \gamma^{\mu} \gamma^{5} \psi,
$$

in standard normalizations [28], with $\kappa^{2}$ the fourdimensional gravitational coupling. Such interaction terms (as well as those derived by integrating out the higherderivative $H$-dependent terms that appear in an effective field theory obtained in the low-energy limit of strings [13]) 
are therefore suppressed by the gravitational coupling. Therefore we may ignore them for our purpose here, which is to discuss the dynamical mass generated by the vector coupling $\tilde{g}_{V}$, which we assume to be hierarchically stronger. ${ }^{5}$

There will also be quantum fluctuations of the D-particles that are independent of their interactions with stringy matter (fermions or photons) and hence of the consequent recoil of the D-particle. Following the example of phonons in solids, which describes the quantum vibrational modes of the lattice ions, we represent the quantum fluctuations of the D-particles by vector Abelian fields, $\mathcal{A}_{\mu}$, which are represented in string language by open strings with their ends attached to the D-particle. Being themselves D-branes, D3-particles can emit such open strings, which thus propagate in the bulk space between two D-particles in the foam. If the D-particles are confined to a single brane world, the field strength of the vector $\mathcal{A}_{\mu}$, which represents the intensity of the low-energy wave emitted by the quantum-fluctuating D-particle, is confined on the brane world, otherwise it propagates in the higher-dimensional bulk. In both cases the open string, whose low-energy vibrational modes contain $\mathcal{A}_{\mu}$, has Neumann boundary conditions over the entire available space. This picture is equivalent to the solid-state picture of phonons, which, though they owe their existence to vibrations of lattice ions, are not confined to the position of the ion but travel through the entire lattice, interacting with the propagating electrons.

The field $\mathcal{A}_{\mu}$ plays the role of a massless Goldstone boson, which is of vector nature as a consequence of the spontaneous breaking of translational invariance by the presence of the D-particles in the D-foam vacuum [8]. In Lorentz-violating field theories, with spontaneous breaking of Lorentz symmetry, the emergence of massless vector Goldstone bosons has been noted in the past, and attempts have been made to identify the photon with such a vector Goldstone boson [29].

We must stress that the vector fields $\mathcal{A}_{\mu}$ should not be confused with ordinary photons $A_{\mu}$, as discussed in [14], which are gauge fields of the electromagnetic $\mathrm{U}(1)$ symmetry in the SM. However, in a similar way to phonons interacting with electrons in a solid, the vector fields $\mathcal{A}_{\mu}$ interact with singlet fermions, and are represented in string language by open strings stretching between the D-particle and the D3-brane world. As in the photonD-particle interaction case discussed above, the recoil of the D-particle during its interaction with the singlet fermion is represented by an antisymmetric tensor field, whereas the quantum vibrations of the D-particle lattice are represented

\footnotetext{
${ }^{5}$ We note for completeness that the four-fermion terms may lead to (positive) vacuum energy contributions of de Sitter type if there is condensation of the corresponding terms involving the chiral fermions. However, Majorana fermions do not lead to such condensates.
}

by the vector field $\mathcal{A}_{\mu}$ that lives in the entire bulk space. In addition, there is an explicit coupling $\tilde{g}_{V}$ of the field $\mathcal{A}_{\mu}$ to the fermion current, similar to the photon-fermion current of charged fermions in QED. From the point of view of the underlying D-brane theory and the associated low-energy type-IIA supergravity action [8], the vector field $\mathcal{A}_{\mu}$ admits an interpretation as a bulk gauge field, with $\mathcal{A}_{\mu}, \mu=0, \ldots 3$ its $(3+1)$-dimensional brane world space-time components. It should be noted that from a string/brane theory point of view, there is no simple non-Abelian enhancement of this gauge field, so its Abelian nature is dictated by the underlying microscopic theory.

We have seen that in homogeneous foam situations the only coupling of the singlet fermion would be through the vector (bulk gauge) field $\mathcal{A}_{\mu}$ :

$$
S_{\psi}=\int d^{4} x \bar{\psi} \gamma^{\mu} i D_{\mu} \psi, \quad D_{\mu}=\partial_{\mu}+i \tilde{g}_{V} \mathcal{A}_{\mu}
$$

where we repeat that $\mathcal{A}_{\mu}$ is not the photon but the vector field representing the quantum fluctuations of the Dparticles, which was absent in the charged fermion case. We recall that in the charged-fermion case of [14] there was also no direct coupling of the charged fermion to the recoil terms, but for a different reason. There, such couplings were identically zero because of the properties of the D-foam, according to which the latter is transparent to charged-fermion fields, because of charge conservation. This argument is strictly speaking valid only for Type IIA string theory D-foam, in which the D-particles are pointlike. For Type IIB string theory D-foam models, on the other hand, D-particles are compactified D3-branes, and as such there are nontrivial, but much more suppressed, fermion-foam couplings [12]. Hence, also in that case the lowest-order (weak-field) effective action term in the fermion sector is given by the QED-like fermion-vector coupling (2.14), where however the photon field of QED is replaced by the vector (bulk gauge) field $\mathcal{A}_{\mu}$.

The effective target-space action on the D3-brane world, where the quantum fluctuating D-particle meets the openstring singlet fermion state, is described by the following Born-Infeld Lagrangian [30,31] augmented with the interaction of the fermion current with the vector (bulk gauge) field $\mathcal{A}_{\mu}$ :

$$
\begin{aligned}
S_{B I}= & \frac{T^{2}}{g_{s}} \int d^{4} x \sqrt{\operatorname{det}\left(g+T^{-1}(B+F)\right)} \\
& +\int d^{4} x \tilde{g}_{V} \mathcal{A}_{\mu} \bar{\psi} \gamma^{\mu} \psi+\int d^{4} x \bar{\psi} i \not \partial \psi,
\end{aligned}
$$

where $F_{\mu \nu}=\partial_{\mu} \mathcal{A}_{\nu}-\partial_{\nu} \mathcal{A}_{\mu}$ is the field strength tensor of the vector field $\mathcal{A}_{\mu}$, and the fermion field $\psi$ represents a massless Majorana spinor, and the coupling $\tilde{g}_{V}$ is discussed below. We remark at this stage that, if photons were to be 
considered, then an additional term involving the Maxwell field strength for the ordinary photons would be present in the argument of the square root of the first term of the action (2.15).

The Lagrangian (2.15) depends on both space-time coordinates and the momentum transfer, and hence cannot be expressed as an ordinary local term in an effective action framework. Nevertheless, as was argued in [14], in the approximation (2.7), in which the D-foam background is treated stochastically, the construction of a low-energy local effective action for the fields $\mathcal{A}_{\mu}$ and $\Psi$ becomes possible, and that action resembles the Lorentz-violating theory appearing in [15].

Let us now return to the effective action (2.15). Defining the generalized field $\mathcal{F}_{\mu \nu} \equiv F_{\mu \nu}+B_{\mu \nu}$, we make use of the fact [32] that in four Minkowski space-time dimensions the determinant $\operatorname{det}_{4}\left(\eta+T^{-1} F\right)$ has special properties that allow the following representation of the Born-Infeld action (we work from now on in units where the string tension is $T=1)^{6}$ :

$$
\begin{aligned}
S_{B I} & =\frac{1}{g_{s}} \int d^{4} x\left(I_{2}+I_{4}\left(1+\mathcal{O}\left(\mathcal{F}^{2}\right)\right)\right)+\text { const } \\
I_{2} & =\frac{1}{4} \mathcal{F}_{\mu \nu} \mathcal{F}^{\mu \nu} \\
I_{4} & =-\frac{1}{8}\left[\mathcal{F}_{\mu \nu} \mathcal{F}^{\nu \rho} \mathcal{F}_{\rho \lambda} \mathcal{F}^{\lambda \mu}-\frac{1}{4}\left(\mathcal{F}_{\mu \nu} \mathcal{F}^{\mu \nu}\right)^{2}\right] .
\end{aligned}
$$

In the weak vector field approximation of interest to us here we can ignore terms of order higher than quadratic in the vector field and the (small) recoil velocity $u_{i}$ field. This is a consistent approximation for relatively heavy D-particles, whose recoil is suppressed by their mass. We also take a quantum average over stochastic fluctuations of the $B$-field, using (2.12), and keep terms quadratic in the vector $(\mathcal{A})$ or (averaged) recoil $(\langle u\rangle)$ fields, including mixed terms of order $\mathcal{A}^{2}\left\langle u^{2}\right\rangle$.

\section{Target-space quantization of the FBI action}

The summation over world-sheet genera leads to targetspace quantization of the Finsler background $B_{0 i}(2.4)$, as discussed in detail in [16]. The properties of the D-particle recoil vertex operator as a (logarithmic) conformal field theory in a $\sigma$-model approach [24] lead, upon summation over world-sheet topologies, to quantum uncertainty relations between the recoil velocities and the collective coordinates describing the initial position of the D-particle,

\footnotetext{
${ }^{6} \mathrm{We}$ remind the reader that the indices are raised and lowered with respect to the background metric $g$, which we assumed to be Minkowski $\eta$. In a general situation, where the metric $g$ is not trivial, the pure foam contributions proportional to various powers of $u_{i}^{2}$ contribute to (dark) vacuum energy [9,11], and can be constrained by cosmological considerations, for instance. We ignore such terms in our discussion here.
}

which correspond to those of canonically quantized collective momentum and position operators for the D-particle in target space. It was therefore argued in [16] that, via (2.7), the summation over world-sheet genera elevates the recoil velocity fields $u_{i}$ into quantum-fluctuating momentum operators in target space ${ }^{7}$ :

$B_{i 0}=u_{i} \Rightarrow \hat{B}_{i 0}=\hat{u}_{i}=-i g_{s} \frac{r_{i}}{M_{s}} \hbar \frac{\partial}{\partial X^{i}} \equiv-i g_{s} \frac{r_{i}}{M_{s}} \hbar \nabla_{i}$,

(no sum over $i=1,2,3$ ).

$B_{i j}=\epsilon_{i j k} u^{k} \Rightarrow \hat{B}_{i j}=\epsilon_{i j k} \hat{u}^{k}$.

Consequently, the uncertainty relations $\left[X^{i}, t\right] \sim u^{i}$ of the classical recoil background [20] are also elevated to quantum operator relations. ${ }^{8}$

There is an important aspect of the correspondence (2.17) that we use in the following. This correspondence was derived from the $\sigma$-model approach to recoil [24], in which one is forced to use time fields $X^{0}$ with the Euclidean signature in order to guarantee the convergence of the world-sheet path integrals. This leads us to extend the correspondence (2.17) to include [14]:

$$
B^{i 0} \Rightarrow \hat{B}^{i 0}=g_{E}^{00} g_{E}^{i k} \hat{B}_{k 0}=+\hat{B}_{i 0},
$$

where the subscript $E$ indicates the Euclidean signature. We revert to the Minkowski signature by analytic continuation only at the end of our computations, after replacing the background $B$ fields with appropriate operators.

In this correspondence (2.17), (2.18), the statistical fluctuations in the foam are implemented by averaging statistically the momentum transfer variable $r(\langle\cdots\rangle)$ over the population of quantum-fluctuating D-particles, using the relations (2.12) for the case of isotropic foam, leading to the correspondence:

$$
\begin{aligned}
\left\langle u_{i}\right\rangle & =0, \\
\left\langle u_{i} u_{j}\right\rangle & =\delta_{i j} \frac{\sigma^{2} g_{s}^{2}}{M_{s}^{2}} p^{k} p_{k} \Rightarrow-\hbar^{2} \frac{\sigma^{2} g_{s}^{2}}{M_{s}^{2}} \delta_{i j} \Delta, \\
\Delta & \equiv \vec{\nabla} \cdot \vec{\nabla} .
\end{aligned}
$$

Via this prescription for first quantization, the effective action (2.16) is mapped onto a particularly simple local effective action, as we now show.

\footnotetext{
${ }^{7}$ The effects of the summation over genera on the fluctuations of the background fields $u_{i}$ have been expressed in closed form only in the bosonic string case. Although closed expressions have not been derived in the world-sheet supersymmetric case [33], we expect our arguments on the correspondence principle (2.17) to characterize all types of string models.

${ }^{8}$ The correspondence (2.17) also leads to master equations that can be used to study the induced decoherence of quantum matter propagating in a quantum-fluctuating D-foam background [26].
} 


\section{A minimal Lorentz-violating QED-like action}

After implementing the correspondences (2.17), (2.18), we obtain the following vector-field-dependent terms in an action of Finsler-Born-Infeld (FBI) type:

$$
\begin{aligned}
S_{B I} \ni & \frac{1}{g_{s}} \int d^{4} x:\left[\frac{1}{4} F_{\mu \nu}\left(1+\frac{b}{16} \hat{B}_{\alpha \beta} \hat{B}^{\alpha \beta}\right) F^{\mu \nu}\right. \\
& \left.+\frac{a}{64} F_{\mu \nu} \hat{B}^{\mu \nu} \hat{B}_{\alpha \beta} F^{\alpha \beta}-\frac{1}{8} F_{\mu \nu} \hat{B}^{\nu \rho} \hat{B}_{\rho \beta} F^{\beta \mu}\right]:+\cdots,
\end{aligned}
$$

where the $\ldots$ in (2.20) represent terms of higher order in the fields $B$ and $F$, and the symbol :...: denotes an appropriate quantum operator ordering, in which the ordering constants $a, b$ obey [14]

$$
a+b=2 .
$$

Terms involving odd powers of the operators $\hat{B}_{\mu \nu}$, vanish in our stochastic Gaussian background (2.19), and so may be ignored. Terms in which the operators $\hat{B}_{0 i}$ or $\hat{B}_{i j}$ lie on the far left-hand-side of the integrand in (2.20) have also been dropped, as they correspond to total spatial derivative terms that do not contribute, under the conventional assumption that the fields decay suitably at spatial infinity on the brane world.

Using as an intermediate step a target-space time with the Euclidean signature when making the correspondence (2.19), as discussed above [see (2.18)], some straightforward algebra using (2.21) reduces (2.20) to [14]

$S_{B I} \ni \frac{1}{g_{s}} \int d^{4} x\left[\frac{1}{4} F_{\mu \nu}\left(1+\frac{1}{4}\left(1-\frac{b}{2}\right) \frac{g_{s}^{2} \sigma^{2}}{M_{s}^{2}} \Delta\right) F^{\mu \nu}\right]+\cdots$,

where the ... represent terms of higher order in derivatives and the Maxwell tensor $F_{\mu \nu}$, and $\Delta$ is the 3-space Laplacian, $\Delta \equiv \nabla_{i} \nabla^{i}=\vec{\nabla} \cdot \vec{\nabla}$.

Whereas the recovery of the FBI action is trivial in the classical limit, quantum ordering ambiguities are an issue. Usually, the quantum ordering of operators is specified by requiring that the effective Lagrangian be Hermitian, which is not an issue here because of the stochasticity of the foamy background (2.19). In principle, is selected by the correct quantum ordering is selected by the full underlying theory of quantum gravity, but this is still elusive, so we proceed phenomenologically. As discussed in detail in [14], the choice $b=2$ would eliminate Lorentz-violating terms, but a solution that leads to dynamical mass generation is preferred, in order to avoid infrared (IR) divergences and the associated instabilities. Assuming that the full quantum gravity theory should act as an IR regulator, one must select an ordering with $b \neq 2$.

Any ordering with $b<2$ would lead to terms that affect the pole structure of the vector propagator in three-space, as the bare propagator of the vector boson $\mathcal{A}_{\mu}$ stemming from (2.22) has the following form [apart from gaugefixing terms that we do not write explicitly here, see the discussion below (3.1) and above (3.9)] [15]:

$$
\begin{aligned}
D_{\mu \nu}^{\mathrm{bare}}(\omega, \vec{p}) & =-\frac{i}{1-p^{2} / M^{2}}\left(\frac{\eta_{\mu \nu}}{-\omega^{2}+p^{2}}-\frac{p_{\mu} p_{\nu}}{\left(-\omega^{2}+p^{2}\right)^{2}}\right), \\
M^{2} & \equiv \frac{4 M_{s}^{2}}{(1-b / 2) g_{s}^{2} \sigma^{2}}>0 .
\end{aligned}
$$

The effective action in Fourier space would therefore no longer be unitary at momenta above the scale $M$. This is exactly what could happen $[11,20]$ if the classical recoil velocity (2.2) were to exceed the speed of light in vacuo, which is unphysical. The low-energy effective action is completely consistent in a classical background, and the mass scale $M$ defines the range of validity of the low-energy local effective action.

In extending our prescription beyond such a classical effective field theory, we look for a choice of quantum ordering that allows the action to be extended beyond the classical limit, so as to describe some aspects of space-time foam for larger momenta. This would provide a partial ultraviolet (UV) completion of the low-energy theory as far as dynamical mass generation is concerned. ${ }^{9}$ Dynamical mass generation is, however, an IR phenomenon and the detailed UV structure of the theory should not affect it. This is, for instance, what happens with the Landau pole of QED, whose presence does not affect dynamical mass generation. ${ }^{10}$ However, our case is different as the effective theory breaks down above a momentum scale where the effective Lagrangian is no longer unitary, because of a change in the sign of the photon propagator. A study of dynamical generation in such a case would need to cut the momentum integrals in the Schwinger-Dyson equations off above the scale $M$. This would be different from the consistent Schwinger-Dyson treatment in [15], which required the cancellation of potential UV divergences, implying implicitly a suitable extension of the model beyond any UV cut-off.

Fortunately, it is possible to avoid such a UV cut-off with a suitable choice of quantum ordering. A minimal class of such orderings, which yield a unitary photon propagator (2.23) in three space dimensions, is that in which the ordering parameter $b$ is in the range:

\footnotetext{
${ }^{9}$ We note that other aspects of the foam, such as vacuum refraction-induced photon delays, cannot be described within the framework of local effective field theories, see [11,20].

${ }^{10}$ There is support for this from lattice calculations but, as far as we are aware, no rigorous proof exists as yet $[34,35]$.
} 


$$
b>2 .
$$

After a formal analytic continuation back to Minkowski space-time, the action (2.22) takes the same form (3.1) as in [15], with the mass scale

$$
M=\frac{M_{s}}{g_{s} \sqrt{\tilde{\sigma}^{2}}}, \quad \tilde{\sigma}^{2} \equiv \sigma^{2} \frac{1}{4}\left|1-\frac{b}{2}\right| .
$$

Any residual ordering ambiguity can be absorbed along with the fluctuations of the foam into a small phenomenological parameter in our first-quantized approach. The remaining ambiguities should be removed when a full, second-quantized quantum gravity model for D-foam fluctuations is developed. ${ }^{11}$ After such a suitable choice of quantum ordering the effective action exhibits maximal suppression of the Lorentz-violating effects and cures IR instabilities through dynamical mass generation.

As already mentioned above and emphasized in $[11,20]$, the phenomenon of induced vacuum refraction for photons cannot be captured by this local effective action. The time delays of photons that are induced by their topologically nontrivial interactions with the D-foam are expected to scale linearly with the photon energy, and thus are suppressed by just a single power of the string mass scale, are purely stringy effects, associated with time-space uncertainties [11,12,20,31] and generated by intermediate (nonlocal) string states stretched between the D3 branes and the D-particles. This is to be contrasted with the modifications due to the foam in the local string effective action (2.22) or (2.15), which are quadratically suppressed by the string scale (2.25), as a result of the stochasticity assumption (2.19).

We now discuss dynamical mass generation for the singlet fermions as a consequence of the dynamics described by the local effective action (2.15).

\section{DYNAMICAL MASS GENERATION FOR SINGLET FERMIONS}

In Ref. [15], dynamical mass generation for fermions has been studied in the context of a $(3+1)$-dimensional QED-like field theory with higher-order spatial derivatives in the photon sector that violated four-dimensional Lorentz symmetry but preserved spatial rotations, and a standard form for the fermion sector. This Lorentz-violating model is not of the Lifshitz type [18], in the sense that there is isotropic scaling between time and space coordinates, but

\footnotetext{
${ }^{11}$ It is interesting to note that for the unique value $b=10$, $\tilde{\sigma}^{2}=\sigma^{2}$, so that the scale $M$ of the Lorentz-violating terms in the action (2.22) is identical to that at which the foam-averaged recoil velocity equals the speed of light in vacuo $[11,20]$. In this case a unique quantum gravity scale would enter the model in different guises.
}

there is a mass scale that suppresses the Lorentz-violating spatial-derivative terms, as we now describe.

\section{A. Review of a minimal Lorentz-violating QED-like model}

The Lorentz-violating Lagrangian considered in [15] is [here the description is adapted so that the $\mathrm{U}(1)$ gauge field $A_{\mu}$ of that work is replaced by the bulk recoil gauge field $\mathcal{A}_{\mu}$, anf the corresponding electron charge $e$ by the vector coupling $\tilde{g}_{V}$ ]:

$$
\begin{aligned}
\mathcal{L}= & -\frac{1}{4} F^{\mu \nu}\left(1-\frac{\Delta}{M^{2}}\right) F_{\mu \nu}-\frac{\xi}{2} \partial_{\mu} \mathcal{A}^{\mu}\left(1-\frac{\Delta}{M^{2}}\right) \partial_{\nu} \mathcal{A}^{\nu} \\
& +i \bar{\psi} \not D \psi,
\end{aligned}
$$

where $\xi$ is a covariant gauge-fixing parameter, $D_{\mu} \equiv \partial_{\mu}+$ $i \tilde{g}_{V} \mathcal{A}_{\mu}$ and $\Delta \equiv \partial_{i} \partial^{i}=\vec{\partial} \cdot \vec{\partial}$, and in our convention the metric is $(-1,1,1,1)$.

For a discussion of the relevance of gauge-fixing terms for the dynamically generated mass and the associated ambiguities, as artifacts of the truncation of the SchwingerDyson equations to one loop, we refer the reader to [14]. In what follows, we use the $\xi=0$ gauge to construct the low-energy interactions of singlet fermions $\psi$ with quantum-fluctuating D-foam in Sec. II D, [cf. (2.22) and (2.14)]. In writing (3.1) we have restricted ourselves to the singlet fermions $\psi$. If the approach is extended to include nonsinglet fermions $\psi_{i}^{\prime}$ in the theory, additional worldvolume gauge fields should be added in the Born-Infeld kinetic terms, and in the covariant derivatives acting on those fermions.

There were no higher-order spatial derivatives for the fermions in [15], so as to avoid nonrenormalizable couplings in the theory of the form

$$
\frac{1}{M^{n-1}} \bar{\psi}(i \vec{D} \cdot \vec{\gamma})^{n} \psi \quad n \geq 2,
$$

and such terms are absent in the framework we introduced in the previous section. Standard $(3+1)$-dimensional QED in a covariant gauge is recovered in the limit $M \rightarrow \infty$, and this scale characterizes the energies at which Lorentzviolating effects become important. It may be the Planck scale, or not, depending on the microscopic origin of Lorentz violation.

The Lorentz-violating terms in (3.2) play a dual role, as discussed in [15]:

(i) First, they introduce a mass scale, $M$, that is needed for generating a fermion mass. Our case corresponds to that of [15] with $\xi=0$, the vector coupling $\tilde{g}_{V}$ replacing the electron charge $e$ and the scale $M$ given by (2.25). That is, the corresponding singlet fermion mass, induced by the interactions with the D-foam, is in our case: 


$$
\begin{aligned}
m_{\mathrm{dyn}} & =\left.M \exp \left(-\frac{2 \pi}{(4+(\xi-1)) \alpha_{V}}\right)\right|_{\xi=0} \\
& =M \exp \left(-\frac{2 \pi}{3 \alpha_{V}}\right),
\end{aligned}
$$

where $\alpha_{V}=\tilde{g}_{V}^{2} / 4 \pi$ is the fine structure constant of our model. ${ }^{12}$

(ii) Secondly, the higher-derivative Lorentz-violating terms provide an effective regularization of the theory, leading to finite gap equations [15]. We emphasize, in order to avoid confusion, that the scale $M$ appearing in this approach does not regulate the theory (3.1), as it regularizes loops with an internal photon line only. Rather, $M$ is a parameter of the model upon which depend physical quantities such as the dynamically generated mass. As we have seen in Sec. II, in our stringy quantum-gravity model that is described by the Lagrangian (3.1) in the lowenergy field-theoretical limit, this scale is expressible in terms of fundamental parameters of the underlying string theory, see (2.25).

The following bare $\mathcal{A}$-gauge field propagator was derived from the Lagrangian (3.1) in [15]:

$$
\begin{aligned}
D_{\mu \nu}^{\mathrm{bare}}(\omega, \vec{p})= & -\frac{i}{1+p^{2} / M^{2}}\left(\frac{\eta_{\mu \nu}}{-\omega^{2}+p^{2}}\right. \\
& \left.+(\xi-1) \frac{p_{\mu} p_{\nu}}{\left(-\omega^{2}+p^{2}\right)^{2}}\right)
\end{aligned}
$$

where $p^{0}=\omega$ and $p^{2}=\vec{p} \cdot \vec{p}$. Note that, since the pole structure is not affected by the Lorentz-violating terms, the $\mathcal{A}$-field remains massless in this minimally Lorentzviolating model [15]. The dynamical mass (3.3) was derived from the following Schwinger-Dyson equation for the fermion propagator (see, e.g., [37]):

$$
S^{-1}-S_{\text {bare }}^{-1}=\int D_{\mu \nu}\left(e \gamma^{\mu}\right) S \Gamma^{\nu},
$$

where $\Gamma^{\nu}$, and $S$, and $D_{\mu \nu}$ are the dressed vertex, the dressed fermion propagator, and the dressed photon propagator,

\footnotetext{
${ }^{12}$ There is an analogy with the magnetic catalysis phenomenon of standard QED [34,36], according to which a sufficiently strong magnetic field catalyzes the dynamical generation of a fermion mass for arbitrarily weak QED couplings. This is an example of Lorentz violation, with the Lorentz symmetry breaking being provided by the direction of the background magnetic field. However, there is an important difference in the magnetic catalysis case from our model (and that of [15]). In the magnetic catalysis case, the magnetic field breaks three-dimensional rotational symmetry and induces an effective dimensional reduction to two dimensions, as a result of the $(1+1)$-dimensional form of the fermion propagator in the lowest Landau level, which is dominant in the strong magnetic field case. Such a phenomenon does not occur here.
}

respectively. The equation (3.5) gives an exact selfconsistent relation between dressed $n$-point functions, and thus is nonperturbative. As a consequence, the would-be divergences are not absorbed by redefinition of the bare parameters in the theory, but the equation is regularized by the scale $M$, which thereby acquires physical significance.

The Schwinger-Dyson equation (3.5) was solved in [15] using the ladder approximation. In this approximation one ignores corrections to the vertex function, which would have led to a system of coupled Schwinger-Dyson equations that would complicate matters significantly. This approximation is known not to be gauge invariant [37], a problem that is generic in off-shell field-theoretical quantities that appear at intermediate stages in calculations of physical on-shell quantities. There are some gauges, termed nonlocal gauges, in which this bare approximation to the vertex is argued to be an exact Ansatz [34]. In our discussion below we restrict our analysis to one loop and pick out the gauge-independent part of the dynamically generated mass in a QED-like theory. In this spirit, loop corrections to the photon propagator fermion wave-function renormalization were neglected in [15], and only the corrections to the electron self-energy were kept.

With our approximations, the dressed fermion propagator can be expressed as

$$
S(\omega, \vec{p})=i \frac{p_{\mu} \gamma^{\mu}-m_{\mathrm{dyn}}}{p_{\mu} p^{\mu}+m_{\mathrm{dyn}}^{2}}
$$

where $m_{\mathrm{dyn}}$ is the fermion dynamical mass, and the Schwinger-Dyson equation (3.5), which involves a convergent integral thanks to the $M$-dependent Lorentzviolating terms, becomes (for $\xi=0$ )

$m_{\mathrm{dyn}}=\frac{\alpha_{V}}{\pi^{2}} \int \frac{d \omega p^{2} d p}{1+p^{2} / M^{2}} \frac{3 m_{\mathrm{dyn}}}{\left(-\omega^{2}+p^{2}\right)\left(-\omega^{2}+p^{2}+m_{\mathrm{dyn}}^{2}\right)}$.

This equation has the obvious solution $m_{\text {dyn }}=0$, but we are interested in a potential second solution, which satisfies the following gap equation that is obtained after integration over the frequency $\omega$ :

$$
\frac{\pi}{3 \alpha_{V}}=\int_{0}^{\infty} \frac{x d x}{1+\mu^{2} x^{2}}\left(1-\frac{x}{\sqrt{1+x^{2}}}\right)
$$

where $\mu \equiv m_{\mathrm{dyn}} / M$ is the dimensionless dynamical mass, which we assume to be small. Both of the terms in parentheses in (3.8) are divergent, but these divergences cancel when combined. As explained in [15], after some approximations valid in the limit $\mu \ll 1$, the nontrivial dynamical fermion mass (3.3) arises as a consistent solution of the equation (3.8). The physical solution of the 
Schwinger-Dyson equation (3.5) is that with the dynamical mass $m_{\mathrm{dyn}} \neq 0$, as it avoids IR instabilities. It is worth noting that the expression (3.3) for $m_{\mathrm{dyn}}$ is not analytic in $\alpha_{V}$, and so can only be found in a nonperturbative approach, such as the Schwinger-Dyson equation used in [15].

Before proceeding, we comment on the $\xi$-dependence of the dynamical mass (3.3). It is an artifact of the oneloop truncation, as we have already mentioned. Indeed, as argued in [14], when one resums appropriate (gaugeinvariant) subsets of Feynman graphs, e.g., using the "pinch technique" that guarantees unitarity [38], there are extra contributions to the dynamical mass, which imply that the gauge-independent physical part of the dynamical mass corresponds to the mass (3.3), except in the $\xi=1$ Feynman gauge, in which all longitudinal parts of the $\mathcal{A}$-gauge-boson propagator vanish. The order of magnitude of the mass in such a case,

$$
\left.m_{\mathrm{dyn}}\right|_{\xi=1}=M \exp \left(-\frac{\pi}{2 \alpha_{V}}\right),
$$

remains the same as in (3.3). For our purposes here, we shall adopt this former result in the $\xi=0$ gauge, since the low-energy string-inspired effective action was constructed in that gauge, as already mentioned. That said, however, we remark that it would be interesting to devise nonperturbative techniques in string theory, e.g., an appropriate version of brane holography, for a nonperturbative computation of the physical dynamical mass.

To recapitulate, in our case, the physical mass of the singlet fermion generated dynamically by its interactions with the foam is:

$$
\begin{aligned}
m_{\mathrm{dyn}} & =M \exp \left(-\frac{2 \pi}{3 \alpha_{V}}\right), \\
\alpha_{V} & =\frac{\tilde{g}_{V}^{2}}{4 \pi}, \quad M=\frac{M_{s}}{g_{s} \sqrt{\tilde{\sigma}^{2}}}, \quad \tilde{\sigma}^{2} \equiv \sigma^{2} \frac{1}{4}\left|1-\frac{b}{2}\right|, \quad b \neq 2 .
\end{aligned}
$$

The magnitude of the coupling $\tilde{g}_{V}$ depends on the string model, and we treat it here as a phenomenological parameter whose estimation we leave to future work. Any $\xi$-gauge dependence between (3.9) and (3.3) can be absorbed formally in its definition, for our purposes, without affecting our order of magnitude estimates.

\section{B. Application to singlet fermions}

If the singlet fermions are right-handed neutrinos, we expect them to be Majorana. Dynamical mass generation for such neutrinos [whose left-handed counterparts belong to $\mathrm{SU}(2)$ doublets of the $\mathrm{SM}$ ] in the above minimal Lorentz-violating framework has been discussed in detail in [19], whose results we borrow for our problem here. As discussed in [19], the relevant low-energy Lorentzviolating Lagrangian is:

$$
\begin{aligned}
\mathcal{L}= & -\frac{1}{4} F_{\mu \nu}\left(1-\frac{\Delta}{M^{2}}\right) F^{\mu \nu}+\bar{N}\left(i \not \partial-\tilde{g}_{V} A\right) \frac{1}{2}\left(1+\gamma_{5}\right) N \\
& +\bar{\nu}\left(i \not \supset-e_{2} \not\right) \frac{1}{2}\left(1-\gamma_{5}\right) \nu
\end{aligned}
$$

where we have concentrated on the interactions of the neutrinos with the foam, ignoring the $\mathrm{SU}(2)$ weak interactions of $\nu_{L}$ for brevity and concreteness. The notations in (3.11) are the following: the Majorana right-handed neutrinos are given by $\nu_{R} \equiv \frac{1}{2}\left(1+\gamma_{5}\right) N$ and the lefthanded neutrinos of the SM sector, belonging to $\mathrm{SU}(2)$ doublets with the ordinary charged leptons, by $\nu_{L}=$ $\frac{1}{2}\left(1-\gamma_{5}\right) \nu$, where $N$ and $\nu$ are four component nonchiral Majorana spinors, with zero bare mass, and for simplicity we restrict ourselves to one generation only for now, commenting later on realistic models with three generations. The coupling $e_{2}$ is either zero (in pointlike D-foam models $[8,11]$ ) or strongly suppressed compared to $\tilde{g}_{V}$ (for Type IIB string D-foam models [12]), by factors that depend on the geometrical characteristics of the foam.

As an example, we consider the Type IIB model of [12], in which our world is viewed as a D7 brane with four dimensions compactified, and the "D-particles" of the foam are represented by compactified D3 branes wrapped around three cycles. Assuming that there is one D-particle per three-volume $V_{A 3}$, and denoting the radius of the fourth space dimension of the D7 brane transverse to the $\mathrm{D} 3$ brane by $R^{\prime}$, this suppression factor is given by

$$
\eta \equiv \frac{e_{2}^{2}}{\tilde{g}_{V}^{2}}=\frac{\left(1.55 \ell_{s}\right)^{4} n_{D}^{(3)}}{R^{\prime}},
$$

where $n_{D}^{(3)}=V_{A 3}^{-1}$ is the D-particle-foam density in threespace. In the model of [12], the values $V_{A 3} \sim\left(10 \ell_{s}\right)^{3}$ and $R^{\prime} \sim 338 \ell_{s}$ were shown to be consistent with phenomenology, but different values are possible in other models. An order of magnitude estimate of $\tilde{g}_{V}$ can be given in that model by noticing [12] that the gauge coupling $g_{37}$ describing interactions of particles on the D7 brane (including singlet fermions) with D-particles (compactified D3 branes) is

$$
g_{37}^{-2}=V g_{7}^{-2},
$$

where $V \equiv V_{A 3} R^{\prime}$ is the volume of the extra four spatial dimensions of D7 branes transverse to the D3 branes, and the coupling $g_{7}^{2} \propto g_{s}$, where $g_{s}$ is the string coupling. The coupling $g_{37}$ (3.13) is identified with the $\tilde{g}_{V}$ coupling in case of singlet fermion excitations, so we have

$$
\tilde{g}_{V} \propto g_{s}^{1 / 2} \sqrt{n_{D}^{(3)} R^{\prime-1}} \mathcal{F}\left(s, t, \alpha^{\prime}\right),
$$

where $s$ and $t$ are appropriate $s$ - or $t$-channel Mandelstam variables in momentum space and $\mathcal{F}\left(s, t, \alpha^{\prime}\right)$ is a momentumdependent form factor associated with string amplitudes 
describing the scattering of such singlet fermionic excitations off D-particles in the model, including string-loop corrections that are suppressed by powers of $g_{s}$ which "renormalizes" the coupling $\tilde{g}_{V}$. This form factor is difficult to compute exactly, given that the target-space action of D-branes is not fully known. For slowly moving excitations with momenta that are small compared to the string mass scale, $M_{s}=1 / \sqrt{\alpha^{\prime}}=1 / \ell_{s}$, for which a field-theoretical approximation is adequate, the form factor is well approximated by unity. However, for higher momenta, and over a wide range of energies, stringy effects of order $\alpha^{\prime}$ become comparable to field-theoretical contributions, as can be seen by rough estimates of form factors in concrete models by applying T-duality arguments, which map the complicated nonperturbative D-brane/string amplitudes into perturbative string-string scattering amplitudes [39]. It is apparent from this discussion that precise estimates of $\tilde{g}_{V}$ depend on the microphysical model, in addition to being proportional to the density of the D-particles in the foam.

Following the detailed discussion in [19], we may regard the initially massless $\nu$ and $N$ as a Majorana doublet $\nu^{M}=$ $(\nu, N)$ that satisfies the Majorana condition $\nu^{M}=\left(\nu^{M}\right)^{c}$, with $c$ denoting the usual charge conjugation operation, and couples to the vector fields. It was shown in [19] by solving the corresponding system of coupled Schwinger-Dyson equations for the fermions $\nu$ and $N$ that, in the single gauge field toy models of relevance here, the only solution for dynamical fermion mass generation is one in which the mass eigenvalues $m_{1}, m_{2}$ are

$$
m_{1}=\lambda_{-}=0 \quad m_{2}=\lambda_{+}=M \exp \left(\frac{-8 \pi^{2}}{3 \tilde{g}_{V}^{2}}\right),
$$

upon fixing the gauge parameter $\zeta=\xi-1$ of [19] to $\zeta=-1$. The mass $m_{1}$ can be identified with the left-handed Majorana mass $M_{L}=0$, and $m_{2}$ [which is identical to $m_{\mathrm{dyn}}$ in (3.10)] is identified with the heavy right-handed Majorana mass $M_{R}$.

There is no nontrivial Dirac mass $\mu$ in the dynamical solution, as explained in [19], but a Dirac mass term can be generated through the usual Yukawa coupling with the Higgs field. The (weak) foam coupling $\tilde{g}_{V} \ll 1$ and the Yukawa coupling to the Higgs field in this scenario can be chosen in such a way that the right-handed neutrino Majorana mass (3.10) is much heavier than the Higgsgenerated Dirac mass, leading naturally to a light active neutrino in the SM sector, as in a conventional seesaw model. In the realistic case of more than one flavor of righthanded neutrino, e.g., three flavors to match the number of active neutrinos as in the $2 M S M$ model [40], one can arrange the active neutrino mass hierarchy by appropriate choice of the Yukawa couplings, even if there is a degeneracy of right-handed Majorana masses (3.10), as in models where there is a universal geometric coupling $\tilde{g}_{V}$.
In other models, $\tilde{g}_{V}$ may be species dependent, which could lead to a mass hierarchy between the right-handed neutrino Majorana masses.

Before closing this section we remark that it is possible to improve the ladder approximation so as to replace the bare coupling $\tilde{g}_{V}$ in (3.10), (3.15) by a running one. This would modify the mass (3.10), but such an improved analysis would not affect significantly the order of magnitude of the nonperturbatively generated mass (3.10). ${ }^{13}$ However, a geometric enhancement of the dynamical mass in multibrane-world scenarios à la Randall-Sundrum [41] was discussed in [14], and is reviewed in the next section.

\section{GEOMETRICAL ENHANCEMENT OF DYNAMICAL FERMION MASSES IN MULTIBRANE-WORLD SCENARIOS}

We now present a geometric mechanism that enhances the dynamical fermion mass (3.10) by a suitable embedding of the model in a higher-dimensional setup involving brane worlds in a Randall and Sundrum (RS) warped bulk geometry [41]. It was suggested in [41] that a large hierarchy between the Planck mass and the $\mathrm{TeV}$ scale could arise in brane-world scenarios in which our world is a negative-tension brane located at a distance $r_{c} \pi$ from a hidden-sector brane embedded in a five-dimensional bulk.

As in RS, we assume that the five-dimensional metric is a solution of Einstein's equations in an anti-de Sitter bulk space with a warp factor of the form

$$
d s^{2}=e^{-\sigma(z)} \eta_{\mu \nu} d x^{\mu} d x^{\nu}+d z^{2},
$$

where $z$ is the bulk (fifth) dimension, and the $x^{\mu}$ are coordinates in our four-dimensional space-time. Because of the warp factor $e^{-\sigma(z)}$ in the metric (4.1), a field on our brane world of mass $m_{0}$ with a canonically normalized kinetic term has a physical mass of the form

$$
m_{\text {phys }}=m_{0} e^{-\sigma\left(z_{i}\right)},
$$

where $z_{i}$ denotes the location of our brane world along the bulk dimension. If $m_{0}$ has a (natural) magnitude around the (reduced) four-dimensional Planck mass $2 \times 10^{18} \mathrm{GeV}$, (4.2) may generate a large hierarchy between the Planck scale and the particle masses $m_{\text {phys }}$ in our world, depending on the size of the exponent $\sigma\left(z_{i}\right)$ in the warp factor. In an RS scenario containing just two branes with opposite

\footnotetext{
${ }^{13}$ The only case where such an analysis enhances significantly the dynamical mass compared to the ladder approximation is magnetic catalysis [34] in the presence of an external magnetic field. However, the reason why the dynamical mass is less suppressed in the improved approximation is the effective dimensional reduction to two dimensions induced by the magnetic field. There is no such a reduction in our Lorentz-violating case, since the singlet fermions are not charged.
} 
tensions, our world is identified with the negative tension brane located at $z_{i}=r_{c} \pi$ with $\sigma=-k|z|, k>0$, and the desired hierarchy is obtained for $k r_{c} \pi \simeq \mathcal{O}(50)$. The exponent $\sigma\left(z_{i}\right)$ is positive in the RS model, so the exponential factor can only decrease the mass with respect to $m_{0}$.

A more complex scenario was proposed in [42], involving many brane worlds and higher-order curvature terms of Gauss-Bonnet type in the bulk:

$$
S=S_{5}+S_{4},
$$

where $S_{5}$ :

$$
\begin{aligned}
S_{5}= & \int d^{5} x \sqrt{-g}\left[-R-\frac{4}{3}\left(\nabla_{\mu} \Phi\right)^{2}+f(\Phi)\left(R^{2}-4 R_{\mu \nu}^{2}+R_{\mu \nu \rho \sigma}^{2}\right)\right. \\
& \left.+\xi(z) e^{\zeta \Phi}+c_{2} f(\Phi)\left(\nabla_{\mu} \Phi\right)^{4}+\cdots\right]
\end{aligned}
$$

where $\Phi(z)$ is the dilaton field and the ... denote other contractions of the four-derivative dilaton terms that are not relevant, since they can be removed by appropriate field redefinitions that leave perturbative string amplitudes invariant. In this case there the bulk Einstein equations have exact solutions in which our world can be identified with a positive-tension brane and, moreover, for the exponent in the warp factor to take negative values, $\sigma\left(z_{i}\right)<0$, which is more important for our purposes as it introduces an inverse RS hierarchy.

The action (4.3), (4.4) is compatible with closed-string amplitude computations in five-dimensional space-times, as needed because we assume closed-string propagation in the bulk. In the stringy case one has [42]

$$
\begin{aligned}
f(\Phi) & =\lambda e^{\theta \Phi}, \quad \lambda=\alpha^{\prime} / 8 g_{s}^{2}>0, \\
c_{2} & =\frac{16}{9} \frac{D-4}{D-2}, \quad \zeta=-\theta=\frac{4}{\sqrt{3(D-2)}},
\end{aligned}
$$

where $\alpha^{\prime}=1 / M_{s}^{2}$ is the Regge slope, $M_{s}$ is the string mass scale, $g_{s}$ is the string coupling and the number of spacetime dimensions is $D=5$.

The four-dimensional part $S_{4}$ of the action (4.3) is given by

$$
S_{4}=\sum_{i} \int d^{4} x \sqrt{-g_{(4)}} e^{\omega \Phi} v\left(z_{i}\right)
$$

where

$$
g_{(4)}^{\mu \nu}=\left(\begin{array}{ll}
g^{\mu \nu}, & \mu, \nu<5 \\
0, & \text { otherwise }
\end{array}\right),
$$

and the sum over $i$ extends over D-branes located at points $z=z_{i}$ along the fifth dimension. Embedding the model (3.1) in such a scenario, we identify (3.1) with the effective four-dimensional field-theory Lagrangian that describes the low-energy dynamics of open strings (representing photons) with their ends attached to our physical world-brane.

The analysis of [42], to which we refer the interested reader for details, has demonstrated that assuming a warped five-dimensional geometry of the form (4.1) there is an exact multibrane solution for the actions (4.3), (4.4), and (4.6) that is depicted in Fig. 2, which involves bulk singularities restricting dynamically the available bulk space. In the bulk regions adjacent to the bulk singularities: $z \sim z_{s}$ the warp factor has a logarithmic solution:
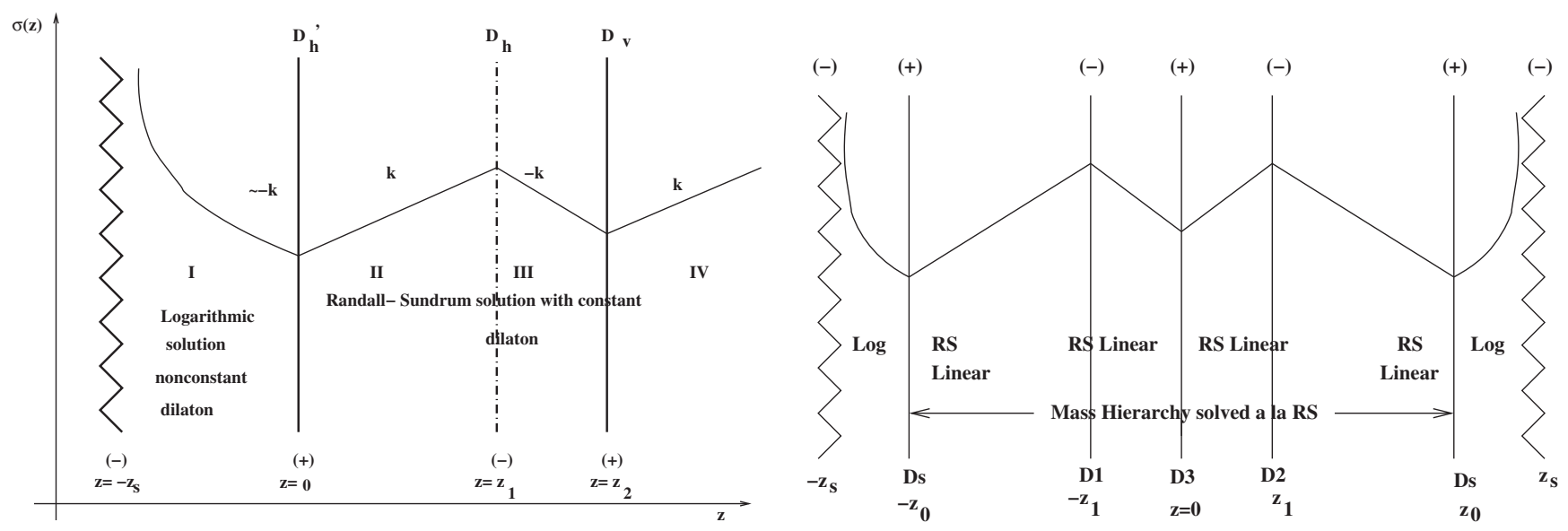

FIG. 2. Left panel: A multibrane scenario in which our world is represented by a positive-tension brane at $z=z_{2}$, accompanied by branes of alternating-sign tensions on the left, which shield a bulk naked singularity that may be thought of as a limiting (singular) case of a negative tension brane. The bulk dimension extends to infinity to the right of the brane world. Right panel: A multibrane scenario in which our world is represented by a positive tension brane at $z=0$, surrounded on both sides by branes with alternating-sign tensions that shield two symmetrically positioned bulk naked singularities. 


$$
\sigma(z)=\sigma_{2}+\sigma_{1} \log \left|z-z_{s}\right|,
$$

while in the other segments of the bulk space the various brane worlds induce linear solutions

$$
\sigma(z)=\sigma_{0}+k z
$$

with the parameter $k$ alternating in sign between adjacent segments of the bulk space, as indicated in Fig. 2. A consistent scenario is obtained by matching the various solutions on each brane.

The detailed analysis of [42] derived solutions of the low-energy gravitational bulk equations with mass hierarchies of the form:

$$
m_{\text {phys }}=m_{0} e^{k\left(2 z_{1}-z_{2}\right)}, \quad k=\sqrt{\frac{2}{3}} g_{s} M_{s}>0,
$$

where $z_{2}$ is the location of our world $\left(z_{2}=0\right.$ in the symmetric scenario shown in the right panel of Fig. 2). The bulk string scale, $M_{s}$, is in general an arbitrary scale in string theory that may be very different from the four-dimensional Planck scale $M_{P}$. In this scenario, however, $M_{P}$ may not be very different from $M_{s}$, e.g., it can be of order [42] $M_{P} \sim M_{s} / \sqrt{g_{s}}$. We stress again that, in these scenarios, our physical world is a positivetension brane.

Clearly it is possible in such a setup to have inverse RS hierarchies, by arranging appropriately the positions of the various branes. For instance, we may have $z_{2}<2 z_{1}$. Identifying $m_{0}$ in (4.10) with our dynamically generated gauge-invariant mass (3.10), the physical mass in our brane world would be:

$$
m_{\mathrm{dyn}}=M \exp \left(-\frac{2 \pi}{3 \alpha_{V}}+\sqrt{\frac{2}{3}} g_{s} M_{s}\left|z_{2}-2 z_{1}\right|\right) .
$$

Singlet fermion masses of the desired phenomenological magnitude can be obtained by arranging appropriately the distance $\left|z_{2}-2 z_{1}\right|$ in the arrangements shown in Fig. 2. ${ }^{14}$

As an indication, if we assume that $g_{s}^{2} / 4 \pi=1 / 20$, a value that is typical in string phenomenology, then the singlet fermion mass due to foam alone would be of order

$$
m_{\mathrm{dyn}}=M e^{-\frac{2 \pi}{3 \alpha_{V}}}=10^{-6.37 / n_{D}} M .
$$

If $n_{D}=\mathcal{O}(1)$ per Planck volume and the mass scale $M$ appearing in (4.11), (4.12) is $\mathcal{O}\left(10^{19}\right) \mathrm{GeV}$, the value of the singlet fermion mass is

$$
m_{\mathrm{dyn}}=\mathcal{O}\left(10^{13}\right) \mathrm{GeV}
$$

\footnotetext{
${ }^{14}$ In the case of the symmetric arrangement in the right panel of Fig. 2, our brane world is located at $z_{2}=0$.
}

This is typical of the masses of singlet right-handed neutrinos that are postulated in seesaw models of light neutrino masses. ${ }^{15}$

\section{CONCLUSIONS AND OUTLOOK}

We have discussed in this paper the possible role of D-foam in generating dynamically masses for singlet fermions. An obvious application is to right-handed neutrinos that then-via a seesaw mechanism-are responsible for generating the small masses of the active neutrinos in the SM. As discussed at the end of the previous section, this possibility is well within the range of uncertainty in the dynamical singlet fermion mass. We note that in this approach Lorentz invariance is violated at the foamy level, but this Lorentz violation is hidden in the effective low-energy field theory, so the phenomenology of neutrino physics at accessible energies resembles that in a conventional seesaw model.

There is considerable flexibility in the magnitude of the singlet fermion mass, which is suppressed hierarchically by the coupling $\alpha_{V}$ whose value is poorly constrained, but may be enhanced hierarchically by geometrical effects in a multibrane-world scenario, as discussed in the previous section. As commented above, we leave to future work the estimation of $\alpha_{V}$ in specific models.

Before closing we recall that the role of D-foam in the dynamical generation of charged fermions was discussed in [14] in a spirit similar to that presented here. However, there are some important differences. In the charged-fermion case, the role of the coupling $\tilde{g}_{V}$ was played by the electrical charge of the fermion, which implied that, for realistic cases, the foam-induced dynamical mass was very much suppressed. However, the dynamical vector field $\mathcal{A}_{\mu}$ discussed here in the singlet neutral fermion case does not couple to a charged fermion, because of charge conservation, and there is no such suppression.

As discussed in the previous section, in addition to the coupling $\alpha_{V}$ to the dynamical vector field $\mathcal{A}_{\mu}$, the singlet fermion mass may be enhanced geometrically in a multibrane-world scenario. This is a possibility also in the charged-fermion case, but realistic masses could be obtained for only one species in that case: the diversity of the masses of the quarks and leptons of the SM could not be obtained via the foam mechanism. On the other hand, this possibility is open in the case of singlet fermions, via appropriate choices of the couplings $\tilde{g}_{V}$ and the geometrical factors, which are species dependent.

\footnotetext{
${ }^{15}$ It is also in the range postulated for the inflaton in a wide range of models of cosmological inflation, so in a supersymmetric extension of our scenario the inflaton could also be identified with the superpartner of the singlet fermion.
} 


\section{ACKNOWLEDGMENTS}

The work of J. E. and N. E. M. is partially supported by the U.K. Science and Technology Facilities Council (STFC) via the Grant No. ST/L000326/1, while that of D. V. N. is partially supported by DOE Grant No. DE-FG02-13ER42020 and in part by the Alexander S. Onassis Public Benefit Foundation.

[1] J. A. Wheeler, Phys. Rev. 97, 511 (1955).

[2] G. Amelino-Camelia, J. R. Ellis, N. E. Mavromatos, and D. V. Nanopoulos, Int. J. Mod. Phys. A 12, 607 (1997); G. Amelino-Camelia, J. R. Ellis, N. E. Mavromatos, D. V. Nanopoulos, and S. Sarkar, Nature (London) 393, 763 (1998).

[3] S. W. Hawking, Commun. Math. Phys. 87, 395 (1982); J. R. Ellis, J. S. Hagelin, D. V. Nanopoulos, and M. Srednicki, Nucl. Phys. B241, 381 (1984).

[4] D. Colladay and V. A. Kostelecky, Phys. Rev. D 55, 6760 (1997); V. A. Kostelecky and S. Samuel, Phys. Rev. D 40, 1886 (1989); V. A. Kostelecky, arXiv:0802.0581, and references therein; J. Bernabéu, F. J. Botella, and M. Nebot, J. High Energy Phys. 06 (2016) 100.

[5] J. Bernabéu, N.E. Mavromatos, and J. Papavassiliou, Phys. Rev. Lett. 92, 131601 (2004); E. Alvarez, J. Bernabéu, and M. Nebot, J. High Energy Phys. 11 (2006) 087; J. Bernabéu, N. E. Mavromatos, and S. Sarkar, Phys. Rev. D 74, 045014 (2006); J. Bernabéu, F. J. Botella, N.E. Mavromatos, and M. Nebot, arXiv:1612.05652; N. E. Mavromatos, Found. Phys. 40, 917 (2010), and references therein.

[6] V. A. Kostelecky and N. Russell, Rev. Mod. Phys. 83, 11 (2011).

[7] J. Ellis, N. E. Mavromatos, and D. V. Nanopoulos, Mod. Phys. Lett. A 31, 1675001 (2016).

[8] J. R. Ellis, N. E. Mavromatos, and D. V. Nanopoulos, Gen. Relativ. Gravit. 32, 127 (2000); Phys. Rev. D 61, 027503 (1999); 62, 084019 (2000).

[9] J. R. Ellis, N.E. Mavromatos, and M. Westmuckett, Phys. Rev. D 70, 044036 (2004); 71, 106006 (2005).

[10] J. R. Ellis, N. E. Mavromatos, and A. S. Sakharov, Astropart. Phys. 20, 669 (2004); J. R. Ellis, N. E. Mavromatos, D. V. Nanopoulos, and A. S. Sakharov, Nature (London) 428, 386 (2004); Int. J. Mod. Phys. A 19, 4413 (2004).

[11] J. R. Ellis, N.E. Mavromatos, and D. V. Nanopoulos, Phys. Lett. B 665, 412 (2008); Int. J. Mod. Phys. A 26, 2243 (2011); Phys. Lett. B 694, 61 (2010).

[12] T. Li, N. E. Mavromatos, D. V. Nanopoulos, and D. Xie, Phys. Lett. B 679, 407 (2009).

[13] J. Polchinski, String Theory, Vols. 1 \& 2 (Cambridge University Press, New York, 2001, 2005), ISBN: 9780511252273 (eBook), 9780521672276 (Print), 9780521633031 (Print) ISBN: 9780511252280 (eBook), 9780521633048 (Print), 9780521672283 (Print).

[14] N. E. Mavromatos, Phys. Rev. D 83, 025018 (2011).

[15] J. Alexandre, arXiv:1009.5834; J. Alexandre and A. Vergou, Phys. Rev. D 83, 125008 (2011).
[16] N. E. Mavromatos and R. J. Szabo, Phys. Rev. D 59, 104018 (1999); see also J. R. Ellis, N. E. Mavromatos, and D. V. Nanopoulos, Mod. Phys. Lett. A 10, 1685 (1995); G. Amelino-Camelia, J. R. Ellis, N. E. Mavromatos, and D. V. Nanopoulos, Mod. Phys. Lett. A 12, 2029 (1997).

[17] See, for instance D. Bao, S.S. Chern, and Z. Shen, An Introduction to Finsler Geometry (Springer-Verlag, New York, 2000); In the context of D-particle foam, such Finsler-type metrics were first derived in J. R. Ellis, N. E. Mavromatos, and D. V. Nanopoulos, Int. J. Mod. Phys. A 13, 1059 (1998); For a short review of this topic, see N. E. Mavromatos, Proc. Sci., QG-PH (2007) 027 [arXiv:0708.2250] and references therein; Finsler metrics have been previously suggested in string theory, but in a different context, see S. I. Vacaru, arXiv:hep-th/0211068 and arXiv:hep-th/0310132; In a field-theoretical context, such metrics have been discussed, among other works, in G. Y. Bogoslovsky, arXiv:0706.2621; SIGMA 4, 045 (2008); G. W. Gibbons, J. Gomis, and C. N. Pope, Phys. Rev. D 76, 081701 (2007); A. P. Kouretsis, M. Stathakopoulos, and P.C. Stavrinos, Phys. Rev. D 79, 104011 (2009); 82, 064035 (2010); Math. Models Methods Appl. Sci. 37, 223 (2014); L. Sindoni, Phys. Rev. D 77, 124009 (2008); M. Anastasiei and S. I. Vacaru, J. Math. Phys. (N.Y.) 50, 013510 (2009). For discussions in the context of generic phenomenological models of nonstandard dispersion relations in quantum gravity and Lorentzviolating Horava-Lifshitz theories; see F. Girelli, S. Liberati, and L. Sindoni, Phys. Rev. D 75, 064015 (2007); J. Magueijo and L. Smolin, Classical Quantum Gravity 21, 1725 (2004); J. Skakala and M. Visser, J. Phys. Conf. Ser. 189, 012037 (2009); S. I. Vacaru, Gen. Relativ. Gravit. 44, 1015 (2012).

[18] P. Horava, Phys. Rev. D 79, 084008 (2009); T. P. Sotiriou, M. Visser, and S. Weinfurtner, Phys. Rev. Lett. 102, 251601 (2009); M. Visser, Phys. Rev. D 80, 025011 (2009) and references therein.

[19] J. Alexandre, J. Leite, and N. E. Mavromatos, Phys. Rev. D 87, 125029 (2013).

[20] For a review on this topic, see N. E. Mavromatos, Int. J. Mod. Phys. A 25, 5409 (2010), and references therein.

[21] N. E. Mavromatos, S. Sarkar, and A. Vergou, Phys. Lett. B 696, 300 (2011); N. E. Mavromatos, V. A. Mitsou, S. Sarkar, and A. Vergou, Eur. Phys. J. C 72, 1956 (2012); N. E. Mavromatos, M. Sakellariadou, and M. F. Yusaf, J. Cosmol. Astropart. Phys. 03 (2013) 015; T. Elghozi, N. E. Mavromatos, M. Sakellariadou, and M. F. Yusaf, J. Cosmol. Astropart. Phys. 02 (2016) 060. 
[22] N. Arkani-Hamed, S. Dimopoulos, G. R. Dvali, and J. March-Russell, Phys. Rev. D 65, 024032 (2001).

[23] I. Antoniadis, E. Kiritsis, J. Rizos, and T. N. Tomaras, Nucl. Phys. B660, 81 (2003).

[24] I. I. Kogan, N. E. Mavromatos, and J. F. Wheater, Phys. Lett. B 387, 483 (1996); J. R. Ellis, N. E. Mavromatos, and D. V. Nanopoulos, Int. J. Mod. Phys. A 13, 1059 (1998).

[25] For a very partial list of works relevant to our discussion here, see V. Gurarie, Nucl. Phys. B410, 535 (1993); J. S. Caux, I. I. Kogan, and A. M. Tsvelik, Nucl. Phys. B466, 444 (1996); I. I. Kogan and N. E. Mavromatos, Phys. Lett. B 375, 111 (1996); M. A. I. Flohr, arXiv:hep-th/0407003; J. Fuchs, S. Hwang, A. M. Semikhatov, and I. Y. Tipunin, Commun. Math. Phys. 247, 713 (2004); M. S. Movahed, M. Saadat, and M. Reza Rahimi Tabar, Nucl. Phys. B707, 405 (2005), and references therein.

[26] N. E. Mavromatos and Sarben Sarkar, Phys. Rev. D 72, 065016 (2005).

[27] A. Hanany and E. Witten, Nucl. Phys. B492, 152 (1997); U. Danielsson, G. Ferretti, and I. R. Klebanov, Phys. Rev. Lett. 79, 1984 (1997).

[28] M. J. Duncan, N. Kaloper, and K. A. Olive, Nucl. Phys. B387, 215 (1992).

[29] For a partial list of references see: J. D. Bjorken, Ann. Phys. (N.Y.) 24, 174 (1963); G. S. Guralnik, Phys. Rev. 136, B1404 (1964); R. N. Sen and C. Weil, Nuovo Cimento A 6 , 581 (1971); A. Kovner and B. Rosenstein, Phys. Rev. D 49, 5571 (1994); P. Kraus and E. T. Tomboulis, Phys. Rev. D 66 , 045015 (2002); J. L. Chkareuli, C. D. Froggatt, R. N. Mohapatra, and H. B. Nielsen, arXiv:hep-th/0412225;
C. A. Escobar and L. F. Urrutia, Phys. Rev. D 92, 025042 (2015).

[30] N. Seiberg and E. Witten, J. High Energy Phys. 09 (1999) 032.

[31] N. Seiberg, L. Susskind, and N. Toumbas, J. High Energy Phys. 06 (2000) 044.

[32] See, for instance, A. A. Tseytlin, arXiv:hep-th/9908105.

[33] N. E. Mavromatos and R. J. Szabo, J. High Energy Phys. 01 (2003) 041; 10 (2001) 027.

[34] V. P. Gusynin, V. A. Miransky, and I. A. Shovkovy, Found. Phys. 30, 349 (2000) and references therein.

[35] See, for example, for a lattice attempt to address this issue: M. Göckeler, R. Horsley, V. Linke, P. Rakow, G. Schierholz, and H. Stüben, Phys. Rev. Lett. 80, 4119 (1998).

[36] V. P. Gusynin, V. A. Miransky, and I. A. Shovkovy, Phys. Rev. D 52, 4747 (1995).

[37] V. A. Miransky, Dynamical Symmetry Breaking in Quantum Field Theories, (World Scientific, Singapore, 1993).

[38] J. M. Cornwall, Phys. Rev. D 26, 1453 (1982); J. M. Cornwall and J. Papavassiliou, Phys. Rev. D 40, 3474 (1989); D. Binosi and J. Papavassiliou, Phys. Rep. 479, 1 (2009).

[39] G. Shiu and L. T. Wang, Phys. Rev. D 69, 126007 (2004).

[40] M. Shaposhnikov, J. High Energy Phys. 08 (2008) 008; J. Phys. Conf. Ser. 39, 176 (2006) and references therein.

[41] L. Randall and R. Sundrum, Phys. Rev. Lett. 83, 3370 (1999).

[42] N. E. Mavromatos and J. Rizos, Int. J. Mod. Phys. A 18, 57 (2003); Phys. Rev. D 62, 124004 (2000). 